\title{
Modelli Europei per un Processo Civile Uniforme*
}

Giuseppe Tarzia

1.- Parlare di modelli europei - e cioè elaborati in Europa a livello sopranazionale - per un processo civile uniforme richiama inmediatamente il pensiero al progetto elaborato tra il 1990 e il 1993 - dopo uno studio preliminare iniziato nel 1988 - da un gruppo di esperti (uno per ciascuno dei dodici Paesi allora facenti parte della Comunità Europea) presieduto dal Prof. Marcel Storme.

Altrettanto immediata è la tentazione di un raffronto col Codigo procesal civil modelo para Iberoamerica ${ }^{l}$; ed il raffronto sembra essere reso ancor più necessario dagli equivoci, nei quali la dottrina internazionale - la non numerosa dottrina, che si è occupata di quello che d'ora in poi chiamerò il progetto Storme - è incorsa nel valutare le finalità, prima ancora che il contenuto di quell'opera.

L'iniziativa infatti si era ufficializzata in contratti con la Commissione ${ }^{2}$, che hanno avuto per oggetto, prima, «uno studio destinato a valutare l'utilità e la realizzabilità»e, poi, l'elaborazione di proposte per «l'approssimazione (non l'unificazione) di quegli aspetti del diritto processuale dei dodici Stati

* Estratto da: Rivista di Diritto Processuale Anno LIV (Seconda serie) - (1999) - N.4

1 Cfr. El codigo procesal civil modelo para Iberoamerica, Texto del Anteprojecto, segunda edición, FCU, Montevideo 1997 e sulle sue fortune Barbosa Moreira, Le codemodèle de procédure civile pour l'Amérique Latine de l'Institute Ibero-Américain de Droit Processuel, in ZZP Int. 3 (1998), p. 437 ss.

2 Richiamo il mio scritto L'Europa del 1993 e il processo civile, nei Rendiconti dell'Istituto Lombardo Accademia di Scienze e Lettere, vol. 124 (1990), p. 79 ss., a p. 83 ss., dal quale sono tratte le frasi riportate nel testo. 
membri della Comunità europea che influiscono direttamente sull'istituzione $e$ il funzionamento del Mercato interno nell'ambito della procedura civile». A questo fine il Gruppo di studio doveva anche stabilire «le priorità da rispettare ... nel contesto del completamento del Mercato interno ... per eliminare le divergenze processuali più importanti e più serie che esistono nei vari settori nei diritti nazionali».

Era chiaro già allora che il mandato, e il conseguente proposito del gruppo di esperti, non era quello di redigere «un codice di procedura civile vero e proprio, un codice completo, magari destinato a sostituire i codici nazionali»: un'opera, questa, che era considerata anche "fuori dalla realtàm. E si avvertiva che "quando si parla, por semplicità, di codice europeo, si vuole indicare la linea di tendenza, la direzione di marcia. Il progetto ... è dunque quello di elaborare un corpus di regole comuni, che dovranno investire non tutte, ma soltanto quelle materie, per le quali il ravvicinamento e l'armonizzazione delle legislazioni processuali civili nazionali possano apparire necessari ai fine dell'istituzione e del buon funzionamento del Mercato unicon.

La necessità di distinguersi dal Codigo modelo, ben noto alla Commissione, era dettata dunque anche dalla impossibilità di predisporre per l'Europa «un Codice tendenzialmente completo, destinato a fornire il modello, appunto, per l'adozione di Codici uniformi»: un disegno si osservava - che "non solo desterebbe resistenze di ogni genere nel l'Europa dei 12 - ove ogni Paese porta seco i frutti di una lunga ed autonoma tradizione giuridica - ma andrebbe al di là dei fini»: i fini, appunto, dettati dall'Atto Unico Europeo del 17 febbraio 1986, circa l'instaurazione del Mercato unico, ossia di «uno spazio senza frontiere interne, nel quale è assicurata la libera circolazione delle merci, delle persone, dei servizi e dei capitalim.

2. - Ho parlato di equivoci, dei quali il gruppo di esperti non si sente responsabile. Già nella presentazione di Storme al volume che raccoglie il progetto ${ }^{3}$ si faceva presente infatti il carattere convenzio-

3 Rapprochement du Droit Judiciaire dans l'Union Européenne, M. Storme. éd, Kluwer, 1997. Sui contributi illustrativi dei giuristi membri del gruppo v. i richiami contenuti nel mio scritto Prospettive di armanizzazione della norme sull'esecuzione forzata nella Comunità economica europea, in Riv, dir. Proc. 1994. p. 207 nota 3; adde Storme, L'unification de la procédure civile en Union Européenne: une chance et un 
nale della denominazione "Commissione Codice giudiziario europeo", data la ben diversa destinazione dello studio, che ho richiamato e che, come è stato da altri notato, doveva costituire semplicemente una bozza per ogni ulteriore approfondimento. Ed infatti esso è redatto in forma di progetto di Direttiva, intitolato al ravvicinamento, o all'approssimaziones del diritto giudiziarío (processuale) nell'Unione Europea.

Nonostante ciò, le puntigliose critiche, che sono pervenute da alcuni studiosi estranei al gruppo di lavoro, hanno avuto per oggetto "la costruzione di un diritto processuale europeo unificato" " o le "proposte della Cornmissione per un codice del processo civile europeo"s.

Non si tratta di una semplice rettifica nominalistica.

L'indagine e il lavoro propositivo del gruppo di esperti sono stati infatti "mirati» a prendere in considerazione soltanto quegli istituti, rispetto ai quali la divergenza delle normative nazionali e le lacune di alcune di esse erano in grado di generare - come è stato ini quella sede ampiamente spiegato - una disparità nella tutela giurisdizionale nei Paesi della Comunità, atta ad influire sul gioco della concorrenza, alterando le regole di un Mercato unico. Questo indirizzo, e questa limitazione, erano del resto coerenti alla fonte che legittimava il lavoro (nel pensiero, allora, della Commissione europea): l'art. $100 \mathrm{~A}$ del trattato CEE, come modificato dall'art. 18 dell'Atto Unico, che abilitava il Consiglio dei Ministri della Comunità, a maggioranza qualificata, ad "adottare le misure relative al ravvicinamento delle disposizioni legislative, regolamentari ed amministrative degli Stati membri che hanno per oggetto l'instaurazione ed il funzionamento del Mercato interno", privilegiando il ricorso allo strumento della direttiva "se l'armonizzazione comporta in uno o più Stati membri una modifica di disposizioni Iegislative».

défit, in Symbolae Vitoldo Broniewicz dedicatae, Lodz 1998, p. 397 ss. e, per un organico esame di questi e di altri interventi che menzionerò in seguito. Normand, Le rapprochement des procédures civiles à I'intèrieur de l'Union européenne et les droits de la défense, in Melanges en l'bonneur de Roger Perrot, Parigi 1996, p. 337 ss. e Il ravvicinamento delle proccedure civili nell'Uniones europea, in Riv. Dir. Proc. 1998, p. 682 ss.

4 Cosi Lindblom, Harmony of the legal spheres, in European Review of Private Law 1997.

5 Così le relazioni alla Tagung dei giuristi tedeschi sul progetto, pubblicate in ZZP 1996, p. 271 ss. 
3.- Opere di dottrina, dunque, sia il Codigo modelo che il progetto Storme; ma opere ispirate a ben diverse finalità, e quindí non comparabíli, né sul piano funzionale né su quello strutturale, né, tanto meno, sul piano ideologico. $E$ certo che il Codigo modelo esprime anzitutto la fiducia nella codificazione, tanto più apprezzabile in quella che è stata realisticamente definita «l'età della decodificazione» ${ }^{6}$. Ma sarebbe arbitrario attribuire agli estensori del progetto Storme un'ideologia opposta. Se si tengono presenti i limiti, che giustificavano il lavoro del gruppo di esperti europeo, si comprende invece l'approccio non sistematico ai temi della disciplina processuale. Non sí è preteso di regolare compiutamente neppure tutti gli istituti presi in considerazione, ma soltanto gli aspetti che ne apparivano più rilevanti ai fini della armonizzazione - non dell'unificazione - dei diritti processuali europei: distinzione, sulla quale, specialmente dopo il recente congresso di Vienna dell'Associazione internazionale di diritto processuale, sarebbe inutile soffermarsi. Altrettanto inutile è segnalare che il lavoro di stesura deg!i articoli, dei consideranda e dei commentari è stato preceduto da un'ampia discussione sulla scelta degli istituti, o dei loro particolari aspetti, da prendere in esame ai fini della proposta di Direttiva.

Non cercherò di riassumere qui le ragioni - esposte in quel documento - che hanno determinato la scelta di ciascuno dei temi raccolti nei 14 capitoli del progetto di Direttiva: la conciliazione; l'inizio della procedura; l'oggetto della lite; la prova, nei suoi aspetti della scoperta dei documenti (discovery), della testimonianza, della riproduzione tecnologica; la desistenza; la contumacia; le spese di giustizia; le misure provvisorie; l'ingiunzione di pagamento; l'esecuzione forzata; l'astreinte; $e$ ancora il computo dei termini, il regime delle nullità e alcune regole reltive alla ricusazione dei giudici e ai mezzi di impugnazione.

Vorrei invece riprendere dal mio scritto deI $1990^{7}$, e completare con qualche richiamo al progetto, la giustificazione, a titolo di esempio, di alcuni soltanto di questi interventi.

4.- Si pensi, anzitutto, all'inizio del processo: alla regola che determina il momento, nel quale il processo si considera iniziato, e quíndi non

6 II riferimento obbligato è al fondamentale contributo di Irti, L'età della decodificazione, Milano, Giuffré 1979.

7 Op. cit., p. 87 ss. 
può essere riproposto, neanche dinanzi ad un giudice di un altro Paese della Comunità. È un problema che la Convenzione di Bruxelles si è posto, stabilendo, nel suo testo originario, che il giudice investito posteriormente della causa deve spogliarsene in favore del primo (art. 21). $\mathrm{Ma}$ la Convenzione non stabilisce quale è il momento in cui il giudice è investito della causa e quindi come si determina quial è il primo e quale il secondo processo; essa rinvia alle legislazioni nazionali, che presentano le soluzioni più svariate. In alcuni Paesi, come in Italia, è necessario, almeno nel processo ordinario, notificare la domanda alla controparte perché la lite sia pendente; in altri la notificazione non è sufficiente, perché la causa deve essere iscritta a ruolo ad iniziativa della parte più diligente. In altri Paesi ancora (o, in altri casi, nello stesso Paese), nei quali prima si deposita il ricorso nella cancelleria del giudice e poi si notifica il ricorso e il decreto di fissazione d'udienza alla controparte, è il momento del deposito quello determinante. Insomma la Convenzione richiama una nozione che ha i significati più vari; e questo può rendere vana la regola e consentire che giudici diversi, applicando il diritto del proprio Stato er definire la pendenza del processo, finiscano per lasciare svolgere contemporaneamente più processi sulla medesima causa. Del resto, la nozione stessa di identità di causa, e quindi di litispendenza, ha assunto notoriamente, nella giurisprudenza Corte della Corte di Giustízia delle Comunità europee, un significato diverso e più ampio di quello della litispendenza inter$\mathrm{na}^{8}$. Questo appariva un altro motivo per un intervento chiarificatore ed armonizzatore, quale si è tentato di fare con l'art. 2.2.2 del progetto. Si è proposto, fondamentalmente, che la litispendenza nasca, nelle procedure che iniziano con domanda presentata in cancelleria, da questo deposito e, nelle procedure avviate con citazione, dalla notiflcazione di quest'ultima: salva la caducazione, con effetto retroattivo, della litispendenza stessa, nel primo caso se la domanda non è notificata al convenuto entro tre mesi dal deposite e, nel secondo caso, se l'iscrizione a ruolo non è compiuta nel termine previsto dalla legge nazionale (che deve dunque prevederlo, restando esclusa una pendenza del processo, in stato di quiescenza, a tempo indefinito).

5.- Si è considerato poi che la domanda produce sempre degli effetti sul diritto fatto valere: per esempio, ne impedisce l'estinzione per 
prescrizione. Quale sia però il momento, nel quale la domanda interrompe la prescrizione del diritto e magari anche la sospende o comunque la rende inoperante per tutto il corso della causa (cfr. artt. 2943 e 2945 c.c. it.), è un problema lontano dal ricevere una soluzione uniforme. In alcuni Paesi è il momento nel quale la domanda è notificata all'avversario; in altri quello in cui la domanda è depositata presso il cancelliere del giudice competente. La conseguenza del permanere di questa disparità comporta che, a seconda dell'Autorità giudiziaria investita (supponiamo che vi sia una pluralità di competenze, di modo che la causa possa essere promossa a Milano o a Londra) il diritto fatto valere continua a prescriversi oppure viene salvato. Ciò contrasta palesemente con quell'esigenza di certezza e di uniformità nel trattamento dei rapporti giuridici, che è alla base di un Mercato unico. Ad eliminare queste incongruenze era diretto l'art. 2.2.1 del progetto, che ricollegava l'effetto interruttivo al deposito della domanda o, rispettivamente, alla notifica della citazione, nei due casi già presi in esame (salva la caducazione retroattiva in caso di inattività), ed inoltre alla richiesta o citazione per una misura cautelare o provvisoria, alla richiesta di ingiunzione di pagamento, alla citazione per la conciliazione, quando è prevista dalla legge.

6.- Un altro setrore, che appariva meritevole di essere «armonizzato", è quello delle misure provvisorie e cautelari e soprattutto, per il loro sviluppo enorme, dei provvedimenti d'urgenza. Anche qui le differenze tra le legislazioni europee sono profonde. In Italia, come sappiamo, per ottenere il provvedimento d'urgenza (art. 700 c.p.c.) occorre dimostrare il pericolo di un danno imminente e irreparabile e il provvedimento deve assicurare provvisoriamente gli effetti della futura decisione di merito: occorrendo, magari anche anticiparli. In Spagna non esisteva, fino al 1984, una misura simile ed è stata allora istituita, ma con molta prudenza, richiedendo un principio di prova scritta del diritto fatto valere. In altri Paesí esistono misure provvisorie che non sono subordinate a questi presupposti e tuttavia differiscono profondamente dai provvedimenti d'urgenza ítaliani, perché, pur avendo, come essi, un contenuto determinato dal giudice a sua discrezione, sono completamente svincolati dal giudizio di merito, che è puramente eventuale. Mi riferisco naturalmente al sistema franco-belga del référé.

Il problema europeo era dunque quello, se si dovesse fissare un nucleo di regole uniformi per questi provvedimenti. II gruppo di esperti ha 
proposto, all'art. 10, una disciplina uniforme delle funzioni di questi provvedimenti, dei loro presupposti, della garanzia del contraddittorio, della competenza, dei mezzi di impugnazione, dei loro effetti, della officacia e della revocabilitá o caducità del provvedimento: una disciplina minima uniforme, salva, come sempre, la specificità ulteriore dei diritti nazionali.

7.- Potrei continuare nell'elencazione e dovrei fario - se ovvie esigenze di brevità non lo impedissero - almeno por le regole sull'astreinte (art. 13) - istituto ancora estraneo, ad esempio, al diritto italiano - per quelle sull'ingiunzione di pagamento (tema sul quale ritornerò tra poco) e por quelle relative all'esecuzione forzata, che miravano allora a porre un minimum di disciplina comune: sia per il momento, nel quale la sentenza diviene esecutiva, sia per le opposizioni all'esecuzione, sia per l'obbligo del debitore di dichiarare gli elementi del suo patrimonio e por il potere degli agenti dell'esecuzione di richiedere a terzi le informazioni relative, sia ancora per agevolare la vendita o cessione forzata dei nuovi beni immateriali (art. 12) ${ }^{9}$.

8.- E noto che il progetto Storme non ha avuto seguito a livello comunitario, per un mutato atteggiamento della Commissiorne: dovuto, a quel che pare, non tanto ad una valutazione del morito (che avrebbe dovuto condurre semmai ad una sua rielaborazione, ma non al uso accantonamento) quanto al principio di sussidiarietà introdotto dal Trattato di Maastricht e alle sue applicazioni, in senso restrittivo, nella formazione della normativa comunitaria.

Eppure credo che, a distanza di oltre sei anni dalla presentazione del progetto (avvenuta nel febbraio 1993), il lavoro svolto possa non apparire vano.

Non è venuto meno, anzitutto, l'interesse per il rapido recupero dei crediti commerciali: un interesse che sollecita interventi a livello convenzionale o comunitario, del quale, come ho già accennato, il progetto Storme si è dato carico.

9.- II quadro delle possibili soluzioni si è qui però allargato con una iniziativa "privata», o meglio corporativa, per un nuovo modello: quello,

9 Sulle prospettive di armonizzazione, in particolare, delle norme dell'esecuzione forzata e sui suoi limiti, dovrei richiamare le mie Prospettive di armonizazione cit. in Riv. Dir. proc. 1994 p. 205 ss. e in Justicia 1993, p. 805 ss. 
cíoè, del titolo esecutivo europeo (presentato sotto l'accattivante sigla T.E.E.), elaborato dall'Union Internationale des Huissiers de Justice e discusso prima, nelle sue linee generali, in un Colloquio a Parigi del giugno 1993 su Les professionels du droit au sein du nouvel espace judiciaire européen $^{10}$; poi (sulla base di Riflessioni introduttive e di un questionario predisposti dal Prof. de Leval), in un convegno tenutosi a Parigi il 20 ottobre 1995, con la presentazione di relazioni e la partecipazione di studiosi per l'Austria, iI Belgio, la Grecia, la Francia, la Germania, l'Olanda, la Spagna, la Svezia e l'Italia: ura larga, anche se non completa rappresentanza dei Paesi ora aderenti all'Unione Europea. In questa occasione l'Union Internationale ha presentato anche un documento sul Titolo Esecutivo Europeo. Il tema è stato ripreso dall'Union in un Colloquio svoltosi a Parigi il 15 e il 16 ottobre $1998^{11}$.

Ricordo brevemente le tappe della "procedura stragiudiziale» descritta nella prima stesura di quel documento, che sembra sviluppare le linee esposte in un progetto di legge presentato al Senato belga nel 1988. La procedura ínízierebbe con una messa in mora, col richiamo della causa del debito e I'indicazione dell'ammontare delle somme reclamate, e con la contestuale domanda di pagamento, notificate dall'ufficiale giudiziario. Si delineerebbe poi un'alternativa: la contestazione nel termine assegnato trarrebbe seco la necessità della procedura davanti al giudice, competente por il merito, del domicilio del debitore; in mancanza di contestazione il credítore potrebbe rivolgersi al giudice competente per l'emanazione del T.E.E.

Non posso nascondere le mie perplessità di fronte a questo modello. Vi è, anzitutto, un problema di rispetto dell'organizzazione delle professioni giudiziarie, come della forma del loro íntervento nelle procedure di recupero dei crediti, che è, attualmente, assai differente nei Paesi dell'Unione Europea. Anche limitandosi a considerare l'ingiunzíone di pagamento, è agevole constatare che le "competenze» che sono attribuite agli huissiers de justice, o ai Rechtspfleger, o forse anche ai cancelliiere in alcuni Paesi, non sono attribuite ai loro colleghi

10 Ricordo, per l'Italia, la relazione di Capponi, Una prospettiva di armonizzazione: il titolo esecutivo curopeo, in Documenti giustizia 1993, p. 1389 ss.

$11 \mathrm{Dal}$ titolo L'Europe judiciaire: Quelle procédure accélérée de recouvrement des créances pour favoriser une efficacité accrue des mesures d'exécution? Gli atti di questo convegno, come di quello del 1995, sono, salvo errore, inediti. 
(dove essi esistono) in altri. D'altro lato, il ruolo degli avvocati, che appare escluso o minoritario, in questa procedura, in alcuni Paesi, è assai rilevante in altri.

II rischio di "conflitti di competenza» tra diversi professionisti nel medesimo Paese dovrebbe accuratamente essere evitato, pur nella finalità, da perseguire, delI'alleggerimento dei compiti del giudice e dello sviluppo della delegazione delle funzioni giudiziarie minori nell'ambito della giurisdizione.

D'altronde, la formazione del titolo esecutivo europeo può essere perseguita più facilmente perr la via di una procedura semplice e poco onerosa, formata su modelli ben conosciuti, quale sarebbe la procedura europea per l'íngiunzione di pagamento, di cui dirò, piuttosto che attraverso una procedura stragiudiziale, fondata sull'idea della provocazione del debitore a contraddire ma al di fuori di ogni investítura del giudice.

L'intervento dell'autorità giudiziaria, senza alcun avvertimento preventivo al debitore, permetterebbe infatti di giungere più rapidamente alla formazione del titolo e più efficacemente alla sua realizzazione con le procedure esecutive. Soprattutto essa vincerebbe più facilmente le resistenze nazionali verso i caratteri essenziali del nuovo titolo esecutivo: la sua validitâ ed efficacia (ottativamente, senza necessità di exequatur) in tutti i Paesi dell'Unione Europea.

10.- In questa situazione continuo a ritenere opportuna (come ha ritenuto il Progetto Storme) l'introduzione di una procedura d'ingiunzione uniforme, valida in tutti i Paesi, a lato delle particolari procedure nazionali: che pure hanno avuto l'avallo della giurisprudenza comunitaria ${ }^{12}$.

Ed infatti le profonde differenze esistenti al riguardo fra i Paesi dell'Unione raccomandano l'istituzione di una procedura uniforme,

$12 \mathrm{Mi}$ riferisco al riconoscimento del valore comunitario dello Zahlungsbefehl tedesco e del decreto ingiuntivo italiano, compiuto dalla Corte di Giustizia delle Comunità Europee, che Ii ha dichiarati, col ricorso. "domand giudiziale o atto equivalente» ai sensi dell'art. 27, punto 2 della Convenzione di Bruxelles (per il riconoscimento) e quindi anche ai sensi dell'art. 34 punto 2 per l'esecuzione. Cfr. rispettivamente C.G.C.E. 16 luglio 1981, in causa 166/80, Klomps c. Michel, in Raccolta di giurisprudenza della Corte 1981,1593, e 13 luglio 1995, Hengst Import BV c. Anna Maria Campase (procedimento C-474/93), in Riv. Dir. Proc. 1996, p. 592 ss., con nota di Giorgetti, Il riconoscimento comunitario del decreto ingiuntivo. 
idonea ad affiancare, dove esistono, le procedure nazionali e a superare i divieti collegati alla necessità di notifica all'estero, fosse pure in altri Paesi dell'Unione ${ }^{13}$.

13 Ricordo la dlversità tra le due versioni che possiamo chiamare, secondo la tradizione dottrinale, dell'ingiunzione pura (il Mahnverfahren tedesco, par. 688 ss. DZPO), e dell'ingiunzione documentale (Mandatsverfabren, $\$ 548$ ss. ÖZPQ; injonction de payer, art. 1405 ss. n. c.p.c. francese, art. 1338 ss. Code judiciaire belga, e titolo X, libro I del c.p.c. del Lussemburgo; ingiunzione, art. 633 ss. c.p.c. Italiano e art. 623 ss. c.p.c. greco) e l'assenza, tuttora, di una procedura siffatta in altri Paesi, come in Spagna, dove se ne propone ora l'introduzione: cfr. Vazquez Sotelo, L'avanprogetto di una nuova legge del processo civile spagnolo, in Riv. dir. proc. 1998, p. 818, a pp. 836-37.

L'ingiunzione documentale è peraltro considerevolmente diversa, nei vari Paesi che l'ammettono:

- sia per quanto riguarda la necessità, o no, di una previa intimazione stragiudiziale di pagamento (prevista, se non erro, solo in Belgio e forse anch'essa all'origine delle scarse fortune del procedimento ingiuntivo in quel Paese);

- sia per quanto riguarda la sua estensione ad ogni credito di danaro o solo a crediti di limitato ammontare (così ancora in Belgio, facendosi riferimento alla competenza del juge de paix) o a creaditi di una ben determinata natura (obbligazioni contrattuali o di origine statutaria e obbligazioni nascenti da alcuni, ma non tutti $\mathrm{i}$ titoli di credi, come in Francia);

- sia per quanto riguarda, infine, la prova del credito.

Si delinea, a quest'ultimo riguardo, la tendenza ad un ampliamento dei documenti giustificativi ammessi, al di là della nozione stretta di "prova scritta" accolta nei codici civili. Ed infatti, se, nel diritto austriaco, rimane ferma l'esigenza che I'ordine di pagamento si fondi su documenti pubblici o documenti privati autenticati $(\$ 548$ ÖZPO) e in altri si richiede la prova per atto pubblico o scrittura privata (art. 623 c.p.c. greco), il Iegislatore belga vuole che la domanda appaia giustificata da «una scritto proveniente dal debitore", che "non deve necessariamente costituire un riconoscimento di debito" (art. 1338 Code judiciare). Più larga è la prassi francese, che ammette fra i documenti giustificativi le fatture e i buoni d'ordine, oltre ai contratti. Per I'Italia basta riferirsi alla nozione allargata di prova scritta, accolta nell'art. 634 c.p.c.

Per quanto riguarda iI secondo aspetto occorre tener presente che non solo l'Italia (art. 633 ultimo comma c.p.c.), ma anche il Belgio (art. 1344 Code Jud.) e la Grecia (art. 624 c.p.c.) non ammettono la pronuncia dell'ingiunzione se la notifica al debitore debba avvenire all'estero (diversamente, facendo eccezione per il caso che la notifica debba aver luogo in uno dei Paesi della Comunità, il par. 688 ZPO tedesca).

Nonostante le pronunce richiamate alla nota precedente, e nonostante la controversia sorta, nella dottrina italiana, sull'attuale vigenza della norma di divieto nei rapporti intracomunitari (cfr. C. Cansolo, La tutela sommaria e la convenzione di Bruxelles: la "circolazione" cumunitaria dei provvedimenti cautelari e dei decreti ingiuntivi, in Riv. 
II testo, elaborato dal gruppo di esperti, deve però essere çonsiderato soltando come un testo di base, che dovrebbe essere migliorato, anzitutto per precisare quale è il document propre à justifier la cause et la quantitè de la créance (art. 11.2). Occorre che si tratti, secondo la formula belga, di un écrit émanant da débiteur sans que soit nècessairemmente requise une reconnaissance de dette oppure si deve ammettere, a livello europeo, e soprattutto per le transazioni commerciali, una nozion più ampia, quale è data, ad esempio,dal diritto italiano?

Considerato che la procedura di ingiunzione non è finora ammessa in tutti i Paesi delI'Unione Europea, crederei prudente scegliere, a livello europeo, il modello più accettabile dell'ingiunzione fondata su documenti (intesa la nozione con qualche rigore) piuttosto che quello dell'ingiunzione assistita soltanto daIl'affermazione del preteso creditore.

Ma, a parte ciò, il Progetto del gruppo di esperti presenta, credo, pregi notevoli, per la precisazione del contenuto della domanda (con indicazione separata del capitale, degli interessi e degli altri accessori: 11.2), per la previsione di un formulario approvato (11.3), per l'avvertimento sui tempi, le forme e il giudice dell'opposizione (11.5), per l'invio al debitore di un formulario per l'opposizione (11.6). Discutibile è incence la brevitá dei termini e sopratttutto l'esonero del debitore inadempiente dalle spese, se paga entro otto giorni.

La Iegge uniforme europea dovrebbe lasciare, a mio avviso, ai legislatori nazionali l'individuazione dell'autorità competente per l'emanazione

Dir. Internaz. Priv e proc. 1991, p. 623; L.G. Radicati di Brozolo, Diritto comunitario e regole processuali interne: inapplicabilità dell'art 633 cod. proc civ., ivi 1993, p. 607 ss.) appare evidente l'opportunità che questa limitazione del procedimento ingiuntivo sia espressamente eliminata, sul piano generale o almeno all'interno dell'Unione Europea. Si pone qui una precisa sollecitazione anche per il legislatore italiano, soprattutto dopo la recente pronuncia della Corte di Giustizia comunitaria (sentenza 22 giugno 1999. C412/97. ED Srl c. Italo Fenocchio) la quale ha statuito:

"1) L'art. 34 deI Trattato CE (divenutto, in seguito a modifica, art. 29 CE) non osta ad una normativa nazionale che non ammette il ricorso al procedimento d'ingiunzione nel caso in cui la notificazione al debitore debba essere effettuata in un Stato membro della Comunità.

2) Una disposizione procedurale nazionale, come quella controversa nella causa principale, non costituisce una restrizione alla libertà dei pagamenti". 
deIl'ingiunzione ${ }^{14}$, come del giudice competente per l'opposizione, risolvendo al riguardo soltanto la questione della giurisdizione e della competenza territoriale a livello europeo. Si pone qui il problema di stabilire se ammettere $\mathrm{i}$ soli dori del domicilio o della residenza del debitore, o anche il foro del Iuogo ín cui l'obbligazione deve essere eseguita. La scelta più restrittiva non solo adeguerebbe il nuovo provvedimento a modelli già ricevuti (come in Belgio e in Francia) ma soprattutto agevolerebbe la difesa del debitore e priverebbe di rilievo la questione della notifica all'estero, eliminando le difficoltà e i ritardi che a questa si ricollegano. Si giustificherebbe anche meglio la regola della libera circolazione, notificazione ed esecuzione dell'ingiunzione in tutti i Paesi dell'Unione Europea senza bisogno di exequatur: regola che si dovrebbe porre per superare anche le condizioni poste dagli artt. 25 ss. della Convenzione di Bruxelles e per assicurare che il nuovo provvedimento vinca la concorrenza con quelli ammessi negli ordinamenti nazionali ed eseguibili ai sensi della Convenzione.

Resterebbe, invece, riservato alla legge uniforme il compito di fissare con precisione i presupposti, il contenuto del ricorso, la natura dei documenti che debbono accompagnarlo, l'efficacia e I'autorità deII'ingiunzione nel caso nel quale il debitore non proponga opposizione nel termine, ed infine gli effetti dell'opposizione.

11.- Un'opera di armonizzazione ${ }^{15}$ è anche resa necessaria, per l'efficacia esecutiva delle sentenze, dal dettato dell'art. $31,1^{\circ}$ comma della Convenzione di Bruxelles, laddove stabilisce che "le decisioni rese in uno Stato contraente e quivi esecutive sono eseguite in un altro Stato contraente dopo essere state munite, su istanza della parte interessata, della formula esecutiva».

II problema del momento, nel quale la sentenza di primo grado diviene esecutiva, appare di centrale importanza per un eguale trattamento delle parti nei vari Paesi, e viva sembra I'esigenza che si eliminino le disparità attuali: esigenza, purtuttavia, trascurata finora, almeno a livello

14 Tenendo anche conto che in alcuni Paesi, come in Svezia, questa non è pronuncciata da un giudíce, ma da altra autoridà (ivi, dall'ufficio dell'ufficiale giudiziario): cfr. Jacobsson, Il processo civile in Svezia, ne La giustizia nei Paesi comunitari, a cura di Fazzalari, vol. secondo, Padova, Cedam, 1966, p. 111 ss. a. p. 127.

15 Riprendo qui osservazioni svolte nello scritto Prospettive di armonizzazione, cit., spec. p. 210 ss. 
comunítario. Ed infatti in alcuni Paesi la sentenza di primo grado non è esecutiva de jure, e l'appello ha pieno effetto sospensivo, salvo che la decisione sia stata dichiarata provvisoriamente esecutiva ${ }^{16}$; in altri ${ }^{17}$ il regime è fondamentalmente il medesimo, ma la parte vittoriosa in primo grado, se anche la sentenza non sia provvisoriamente esecutiva, può ottenere sequestro conservativo a garanzia della futura esecuzione; altrove $^{18}$, I'esecuzione provvisoria delle sentenze di primo grado appellate è consentita entro limiti fissati dalla legge e rimessa alla valutazione del giudice, solo contro costituzioone di una cauzione. In Italia, la legge 26 novembre 1990, n. 353 di riforma del processo civile ha sovvertito la regola tradizionale, derivante dalla codificazione napoleonica ed ha introdotto il principio dell'efficacia immediatamente esecutiva di tutte le sentenze di primo grado. Il medesimo principio vale, se non erro, nei Paesi di common law.

Evidente appare la necessità dell'introduzione di una regola uniforme, ad evitare una protezione diseguale del credito e fenomeni possibili dii forum shopping da parte del debitore: che, promuovendo un 'azione di accertamento negativo del credito, darebbe luogo alIa Iitispendenza, anche per la successiva azione di condanna del creditore, ai sensi dell'art. 21 della Convenzione di Bruxelles e dell'ampia nozione di litispendenza internazionale, accolta dalla Corte di Giustizia delle Comunità Europee ${ }^{19}$.

12.- Si deve dare atto, da ultimo (anzi, da penultimo), che, sulla basee dei lavori di alcuni "Comitati di saggi" costituiti presso la Commissione Europea nel 1996 e nel $1997^{20}$ la Commissione ha

16 Così in Francia, art. 514 ss., 524, 539 nuovo c.p.c.

17 Come in Belgio, art. 1397 ss, e 1414 Code judiciaire. Simile è la soluzione tedesca: il pignoramento per crediti di danoro è ammesso, sulla base della sentenza di primo grado, ma non si può procedere alla vendita se non è prestata cauzione (par. 720 a DZPO).

18 Come in Spagna, art. 385 LEC.

19 Cfr. Kerameus, Problemi attuali della litispendenza internazionale nel processo civile, in Riv. dir. Proc. 1990, p. 1001 ss.; Di Blase, Connessione e litispendenza nella convenzione di Bruxelles, Padova 1993.

$20 \grave{E}$ opportuno segnalare che, nel corso di essi, I'Unione internationale des huissiers de justice ha presentato, sulla materia dell'ingiunzione, ed a seguito delle obiezioni sollevate in varie sedi contro il progetto di T.E.E., anche due progetti alternativi, che prevedono, il primo, lingiunzione di pagamento con controllo giudiziario a priori e, il 
presentato una comunicazione al Consiglio e al Parlamento Europeo intitolata "Verso una maggiore efficienza nell'ottenimento e nell'esecuzione delle decisioni nell'ambito deIl'Unione europea” ${ }^{21}$, assieme ad una proposta di modifica della Convenzione di Bruxelles del $1968\left(98(\mathrm{C} 33 / 05)^{22}\right.$. E questo pare oggi iI punto di partenza per dare nuovo impulso al progetto di creazione progressiva di un processo civile uniforme europeo; iniziando da interventi sul testo fondamentale in materia e cioè, appunto, dalla convenzione di Bruxelles deI 1968, cui si sono aggiunte recentemente le Convenzioni europee firmate a Bruxelles il 23 novembre 1995 sulle procedure di insolvenza e il 26 maggio 1997, sulla notificazione degli atti giudiziari ed extragiudiziali e soprattutto la Convenzione del 28 maggio 1998 - c.d. Bruxelles II - sulla competenza, il riconoscimento e l'esecuzione delle decisioni nelle cause matrimoniali ${ }^{23}$.

Senza poter riassumere qui compiutamente le statuizioni suggerite dalla Commissione e, meno ancora, la larga motivazione che ne è stata data, noterò che si è soprattutto proposto:

I. come criterio base della competenza (o, noi diremmo, dei limiti territoriali della giurisdizione), la sostituzione del domicilio con la residenza abituale (art. 2 Conv.); la revisione delle norme sulle competenze speciali, ivi comprese quelle in materia di assicurazioni e di contratti conclusi dai consumatori, e di quelle sulle competenze esclusive e sulla proroga di competenza;

II. una nova regola di competenza (ex art. 18 bis, $1^{\circ}$ comma), per le misure cautelari e provvisorie, a favore dello Stato sul territorio del quale queste misure possono essere effettivamente eseguite, sostituendo l'art. 24 della Convenzione;

secondo, I'ingiunzione di pagamento con controllo giudiziario a posteriori, che sarebbe limitata alla tutela dei crediti professionali. La materia, come si vede, è lungi dall'aver raggiunto un adeguato assestamento, v.g. indice di consenso, a livello comunitario e interprofessionale.

21 (98/C33/03) pubblicata sulla Gazz. aff C.E. 31 gennaio 1998.

22 Intitolata Proposta di atto del Consiglio che stabilisce la convenzione relativa alla competenza giurisdizionale, al riconoscimento e all'esecuzione, negli Stati membri dell'Unione europea, delle decisioni in materia civile e commerciale.

23 Si possono leggere queste tre Convenzioni, in Riv. dir. internaz. Priv. e proc., rispettivamente 1996, p. 661 ss.; 1998. p. 668 ss. e 1998, p. 928 ss, 
III. I'introduzione, nella Convenzione, di nozioni di diritto uniforme in materia di misuee provvisorie e cautelari (se ne veda la definizione nell'art. A 8 bis $2^{\circ}$ comma) e di litispendenza (definizione del momento nel quale il giudice si considera adito, art. $21,3^{\circ}$ comma);

IV. l'agevolazione della circolazione delle sentenze in Europa, tramite una revisione delle disposizioni sul riconoscimento e sull'esecuzione contenute nelle Convenzioni di Bruxelles e di Lugano, fondata sulla tecnica dell'inversione del contenzioso e sulla limitazione dei motivi di rifiuto del riconoscimento e dell'esecuzione (nuovi artt. 27, 31, 34, 36, 37 bis e ter e 38);

V. l'idonneità della sentenza di condanna, anche prima dell'exequatur, a consentire misure provvisorie e cautelari in qualunque Stato contraente, secondo la legislazione di questo Stato (nuovo art. 27 (Conv.).

Inoltre si pone allo studio ${ }^{24}$ :

l'opportunità di creare un titolo esecutivo europeo, che possa beneflciare di un exequatur quasi automatico (recuperando, così, almeno in parte, i suggerimenti degli Huissiers);

- L'adozione di misure al fine di assicurare la trasparenza del patrimonio (come l'obbligo di dichiarazione del patrimonio da parte del debitore esecutato) e lo scambio di informazioni tra le autorità esecutive;

- P'introduzioonee di una procedura rapida por il recupero dei crediti di danaro e di regole sul pignoramento dei conti bancari.

13.- A queste e ad altre proposte, che sono state elaborate a livello dottrinale - come quella della creazione di una misura cautelare euopea - si aggiungono ora le precise indicazioni del Trattato di Amsterdam del 2 ottobre 1997, entrato in vigore il $1^{\circ}$ maggio 1999. Ed ínfatti, a modifica ed integrazione del Trattato di Maastricht 7 febbraio 1992 sull'Unione Europea, è ivi previsto (art. $73 \mathrm{I}$, introdotto nel predetto Trattato fondamentale dall'art. 15 del Trattato più recente) che "allo scopo di istituire progressivamente uno spazio di libertà, sicurezza e giustizia, il Consiglio adotta: ... c) misure nel settore della cooperazione giudiziaria in materia civile, come previsto all'articolo $73 \mathrm{M}$ \%. Queste misure, testualmente (art. $73 \mathrm{M}$ ) includono: 
in primo luogo, "II miglioramento e la semplificazione: - del sistema per la notificazione transnazionale degli atti giudiziari ed extragiudiziali; - della cooperazione nell'assunzione dei mezzi di prova;- del riconoscimento $e$ dell'esecuzione delle decisioni in materia civile e commerciale, comprese le decisioni extragiudiziali»;

in secondo luogo, "la promozione della compatibilità delle regole applicabili negli Stati membri ai conflitti di leggi e di competenza giurisdizionales;

da ultimo, "I'eliminazione degli ostacoli al corretto svoIgimento dei procedimenti civili, se necessario promuovendo (a compatibilità delle norme di procedura civile applicabili negli Stati membri».

$\mathrm{Ci}$ troviamo quindi di fronte ad un programma a vasto raggio, nel quale la ripresa dei modelli già elaborati - sia pure con ogni opportuna loro valutazione critica - dovrà essere messa nell'agenda delle Autorità europee. L'ultima, soprattutto, delle previsioni ora menzionate lascia un'ampia discrezionalità nella scelta, necessariamente progressiva, dei settori di intervento: una discrezionalità nella quale v'è da augurarsi che la Commissione si avvalga, riprendendo in esame la maggior parte dei modelli sin qui proposti, pur con ulteriori integrazioni $i^{25}$.

La consapevolezza che il cammino verso un processo civile uniforme - nel senso più lato dell'espressione - è lungo e difficile, anche nel solo ambito dell'Unione Europea, non deve dunque indurre allo scetticismo o, peggio, alla rinuncia.

25 II grande ostacolo, che sembra comunque destinato a restare, sul terreno dell'esecuzione forzata per crediti di danaro, è dato dalla contrapposizione tra il principio della par condicio creditorum e quello di priorità del primo pignorante. Rinvio, per una ricognizione a livello europeo, sul primo problema al mio scritto La distribution des deniers dans, l'exècution forcée en Europe, in Revue internationale de droit comparé 1999, p.331 ss., e, per alcune precedenti considerazioni de jure europaeo condendo, alle Prospettive di armonizzazione, cit., pp. 218, 219. Per a regola saisie sur saisie ne vaut, richiamo le considerazioni svolte nel mio lavoro Les titres exfecutoires et le recouvrement des créances dans l'Union Européenne, in Actualités du droit (Revue de la Faculté de Droit de Liège), 1995-2, p. 382 ss. 